\title{
Construction of Big Database in the Field of Public Security: A Case Study in Shenzhen
}

\author{
Jixiao YIN", Diping YUAN ${ }^{\mathrm{a}, 1}$, Guohua WU, ${ }^{\mathrm{a}, \mathrm{c}, \mathrm{d}}$, Zuoxin WANG ${ }^{\mathrm{a}}$, Yiping ZENG ${ }^{\mathrm{b}}$, Jun \\ $\mathrm{LING}^{\mathrm{a}}$ and Qinliang $\mathrm{LI}^{\mathrm{a}}$ \\ ${ }^{a}$ Shenzhen Urban Public Safety and Technology Institute, Shenzhen 518000, China \\ ${ }^{\mathrm{b}}$ Southern University of Science and Technology, Shenzhen 518000, China \\ ${ }^{\mathrm{c}}$ Harbin Institute of Technology, Shenzhen 518000, China \\ d Shenzhen Urban Transport Planning Center Co. Ltd, Shenzhen 518000, China
}

\begin{abstract}
In terms of the actual needs of the emergency management department, at the beginning of the establishment of Shenzhen Emergency Management Bureau, all kinds of information resources have not been connected, and the business of the Department is still supported by the information assets of the original unit of the transferred department. However, there are significant differences in the construction years, construction standards and system architecture of the existing information systems, which lead to the "one number, multiple sources" and multiple management of all kinds of business data, and the systems are basically in the chimney construction pattern of mutual separation. At the same time, the exchange and sharing of internal and external data of the Emergency Management Bureau lacks a unified data standard, and a unified data platform has not been built for centralized management. Therefore, it is difficult for the newly established emergency management bureau to effectively support the business process reengineering, collaborative command, big data intelligent decision-making and other needs. In addition, considering the construction of smart city at the municipal and district levels, as well as the complex information situation such as the information collaboration within the security committee office and emergency committee system, it is urgent to prepare the top-level design of emergency management information, and plan the future development with a higher position, a wider vision and a new idea.
\end{abstract}

Keywords. Emergency management, data platform, big data intelligent decisionmaking

\section{Introduction}

In March 2018, the first session of the 13th National People's Congress approved the institutional reform of the State Council to establish the Emergency Management Department of the PRC. As an important function, emergency management should be comprehensively strengthened by incorporating into the economic and social development, establishing an organizational system to improve the emergency

${ }^{1}$ Corresponding Author, Diping YUAN, Shenzhen Urban Public Safety and Technology Institute, Shenzhen 518000, China; Email: yuandp@szsti.org. 
prevention, early warning and disposal as well as strengthen daily prevention, emergency preparedness and unified scheduling [1-2]. The series of measures can not only reduce the risk of the disaster and losses but also save lives effectively [3-5].

In order to strengthen the level of local emergency management informatization, the Ministry of Emergency Management has successively issued the "2019 Local Emergency Management Informatization Implementation Guide", "Emergency Management Informatization 2019 First Batch of Local Construction Tasks", "Emergency Management Technology Information Notice on the First Batch of Standards and Normative Document Catalogues", and jointly issued the "Notice on Strengthening the Management of Basic Emergency Response Information" with the Office of the State Council's Security Committee and the Office of the National Disaster Reduction Commission. These documents make clear the relevant requirements, key tasks and standards of emergency management technology informatization construction, and put forward clear development ideas and requirements for the development of emergency informatization in Shenzhen [6].

Based on the construction requirements of the 2019 Data Governance System Local Construction Tasks of the Ministry of Emergency Management, Shenzhen emergency management bureau carries out the overall design and planning in combination with the actual situation of the city, and fully evaluates the data demand, functional demand, technical demand and other needs. After that, Shenzhen emergency management bureau builds the big database, which includes six key contents: data standards and specifications, emergency management business database, emergency management spatio-temporal information database, big data governance system, big data application support, and artificial intelligence enabling service. It can realize the orderly sharing and moderate opening of emergency data, deepen the application of emergency big data, promote the development of new formats, support the modernization of emergency management system and governance capacity, and provide standard and unified data support for Shenzhen emergency management [7-9].

\section{Overall Structure}

The structure of Shenzhen emergency management big data governance system is shown in figure 1 below. Big data basic platform layer: a big data framework based on Shenzhen government cloud, including all kinds of cloud infrastructure, distributed computing and storage and other related components. Parallel computing and distributed storage are built on the basis of open-source big data platform Hadoop. They are enhanced in functionality, reliability, ease of use, maintenance, security and other aspects to form an enterprise level Hadoop platform. Based on shared nothing/MPP architecture, distributed parallel database is oriented to open arm and x86 platforms. Data is evenly distributed across all nodes, and all nodes work in parallel.

Data access: data access defines the process, method and flow mechanism of data acquisition, processing, governance and organization in the initial stage according to the business requirements of emergency management. In addition, according to data exploration and definition, it connects multi-source heterogeneous data to big data center and completes data reconciliation with data provider.

Data processing: data processing is based on the data definition of data access link, aiming at the big data characteristics of emergency management data, such as huge scale, diverse types, high-speed flow, complex and changeable, uneven quality, and different 
value density.

Data management and control: data management and control are mainly the process control and quality supervision of the whole life cycle of emergency data resources, which includes clarifying data assets, improving data quality, ensuring the safe use of data and promoting data circulation. According to the sensitivity of data content, data resources are classified. Through standardized data governance, it can realize the transparency, manageability and controllability of emergency data resources, improve the implementation of data standards and promote data circulation and value extraction.

Data sharing and exchange: data sharing and exchange realize internal and external data exchange of Emergency Management Bureau through library table, file, service interface and so on [10]. Data exchange relates to municipal government information resource sharing and exchange platform to obtain data related to emergency management business of departments at the same level. At the same time, it relates to provincial data sharing and exchange system to realize data sharing and exchange at provincial and municipal levels.

Big data resource pool: big data resource pool is a data resource pool, which composed of original database, resource database, subject database and special database after the whole process of data governance, such as data access, data processing, data control, data sharing and exchange.

Data service: data service is to extract data from the original database, resource database, theme database and thematic database of big data resource pool and carry out relevant data processing and interface encapsulation. It provides data support services for smart application of emergency management as well as meet the data needs of various departments, centers and institutions in various application constructions. The construction of data service layer mainly includes: query and retrieval service, data authorization service, data visualization and so on.

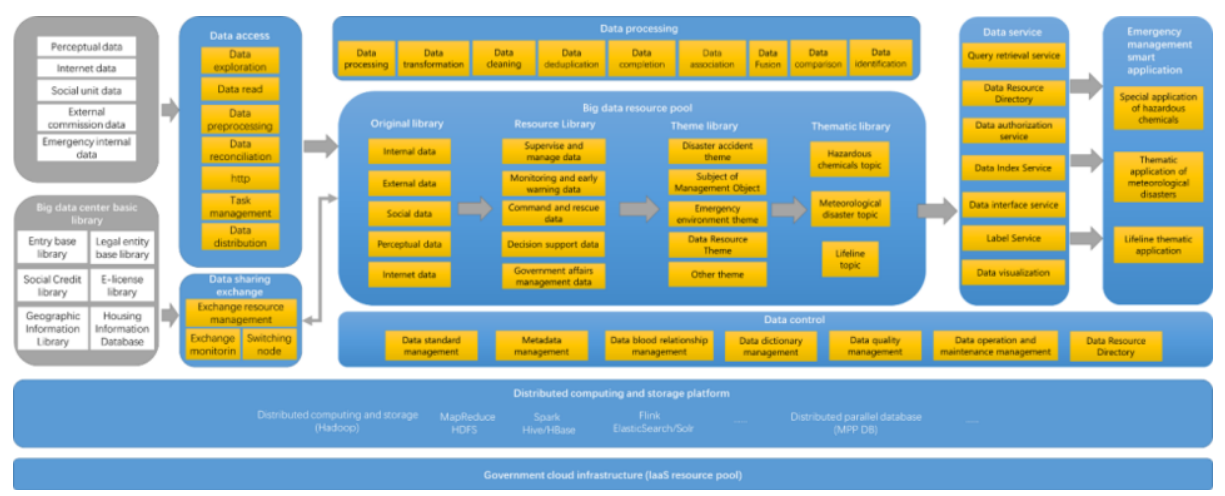

Figure 1. Overall framework of emergency management data governance.

\section{Technical Characteristics}

\subsection{Unified Storage}

Shenzhen emergency management big data governance system realizes a unified storage platform to meet the data access needs of multiple types of data and services. The storage platform not only supports block, file, object and HDFS protocol to meet the access of 
different computing nodes, but also supports flexible deployment and large-scale expansion of multiple data types.

\subsection{Big Data Technology}

Based on Hadoop organization, the data support system adopts distributed data processing technology, which provides massive data storage, analysis and query and realtime streaming data processing and analysis capabilities. Following the latest technology of Hadoop open-source community and maintaining the openness completely, it does not use private architecture and components and integrates the latest components. Moreover, it makes enterprise level enhancement and continuous improvement in reliability, safety, management and other aspects, and integrates with products of all parties in big data center through standard northbound interface.

\subsection{Artificial Intelligence}

AI scheduling platform integrates the major features of machine learning, deep learning, multi-algorithm management and heterogeneous resource scheduling. It provides onestop data preprocessing, feature engineering, model development, model training, and model reasoning service publishing end-to-end capabilities, so that AI business can be quickly developed by reducing the threshold of AI application. All in all, it can provide the AI services such as image recognition, face recognition, intelligent robot and so on for customers.

\subsection{Safety Self-Control}

In the context of trade conflict between China and the United States, it is particularly important to maintain a stable supply of core devices and independent intellectual property rights of products. The construction and deployment of big data platform is based on ARM server, rather than Intel x86. The key chips such as processor, storage, network IO and management of slave server are independently developed by Chinese companies, and will not lead to the risk of chip supply due to the change of international situation. In addition, the big data platform software is a non-OEM product with completely independent intellectual property rights.

\subsection{Distributed Parallel Database}

Adopting massive parallel processing framework, big data platform is equipped with enterprise level large-scale parallel processing relational database, which provides $\mathrm{Pb}$ level data processing capacity by row storage and column storage. According to memory analysis technology, it can query the detailed business of security, telecommunications, finance, Internet of things and other industries. It can not only efficiently process 10 billion row multi table join queries, but also online transaction processing and online analytical processing.

\subsection{Separate Storage and Computing}

Adopting the separation technology of storage and computing, the big data platform 
solve the adaptability of storage architecture in the emergency data management system and provides a unified data storage base for multi cluster of big data, which improves the utilization of big data storage resources. When the CPU resource is insufficient, the computing service node is expanded and when the storage resource is insufficient, the storage service node is expanded. In addition, it realizes data lifecycle management of big data cluster by automatically deleting expired data. What's more, it can not only store a variety of data types, such as remote sensing, video, pictures and maps, but also access a variety of applications, such as big data, cloud services, and GIS.

\section{Overall Data Flow}

The overall data flow of Shenzhen emergency management big data governance system is shown in figure 2. Application support layer provides general support, algorithm model support and Internet of things real-time data access for big data governance system and data support layer while data support layer provides data support for application support layer. In addition, big data governance system and data support layer provide data support and services for business application systems. Firstly, the real-time raw data of safety production, urban safety, natural disaster and emergency scene perception access to Internet of things platform and then access to the data resource pool through the data management system.

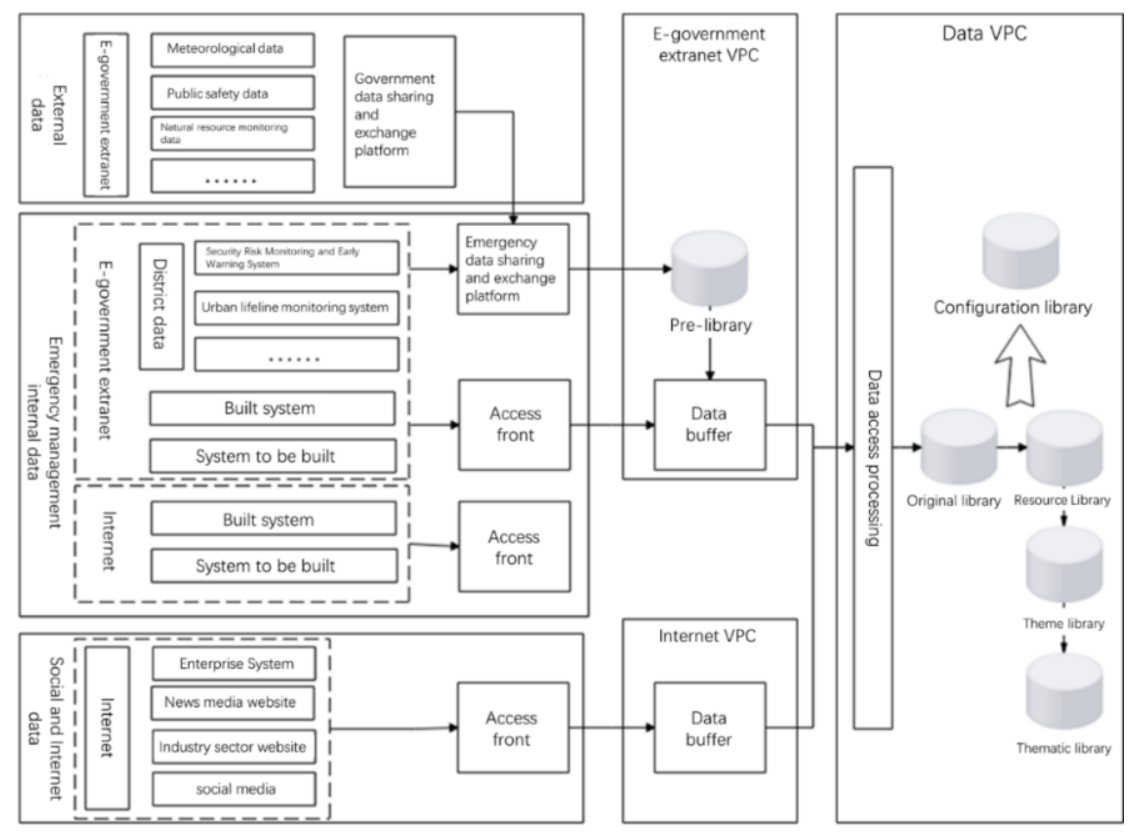

Figure 2. Data flow structure of the governance system.

Firstly, the system integrates data into the original database from various sources and formats, such as internal and external data of Emergency Management Bureau, business system data of emergency management department, distributed data, social unit data, Internet crawler data and scattered data. Then, according to the standard of data 
exchange and data format, resource database is formed by fuse the data of each sink and merge the same object data of different data sets. Finally, theme library and configuration library are formed through assessing requirements of data organization and business.

\section{Platform Realization and Application}

Researching and developing Shenzhen Public Security big data management and exchange sharing platform, establishing open, shared, integrated and associated data management mode, form public security big data management standard system, and provide data support for the construction of urban public security comprehensive situation visualization system.

\subsection{Data Access and Open, Shared, Integrated and Associated Data Management Mode}

Data in 15 safety committee members and 6 emergency business systems in Shenzhen has accessed, with a total of 871 data tables, 224 million pieces of data and 141 GB data volume, realizing the data access and aggregation of safety committee members including meteorological bureau, market supervision and Administration Bureau, Water Affairs Bureau, housing construction bureau and gas group. At the same time, access to safety production, three prevention, urban lifeline, geological disasters, risk assessment and other five types of emergency management business data.

\subsection{Building Public Security Information Resources Service Capacity}

The data governance architecture is shown in Figure 3. At present, we have completed the construction of the original database of production safety data, urban safety field data and comprehensive management data, and formed various subject databases and special subject databases, with 534 governance data tables and 130 million governance data. We have also formulated resource directory and developed 265 data interfaces, realizing hierarchical user access control and automatic data on-demand backflow mechanism, to provide data support for the comprehensive platform of urban public security spatiotemporal big data risk prevention and control and units at all levels.

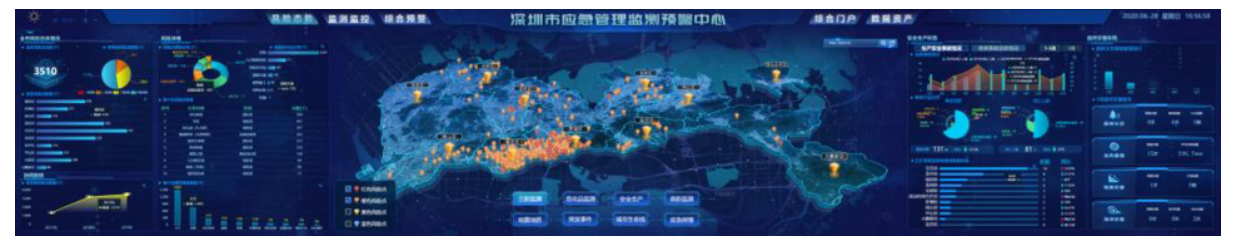

Figure 3. Shenzhen Public Security big data management and exchange sharing platform.

\section{Conclusion}

This project develops a public security big data management and exchange sharing platform, establishes an open, shared, integrated and associated data management mode, 
forms a public security big data management standard system, and provides data support for the construction of urban public security comprehensive situation visualization system. It has access to the data of 15 safety committee members and 6 emergency business systems in Shenzhen, with a total of 871 data tables, 224 million pieces of data and $141 \mathrm{~GB}$ of data. It has achieved the data access and aggregation of safety committee members including meteorological bureau, market supervision and Administration Bureau, Water Affairs Bureau, housing and Construction Bureau, gas group, etc., and is providing support to the relevant departments and departments directly under 15 cities Each district Emergency Management Bureau provides services, initially realizes the dynamic public security risk management and control, intelligent monitoring and early warning, and effectively supports the development of emergency management informatization in Shenzhen.

\section{Acknowledgments}

This research was supported by National Key R\&D Program of China (2019YFC0810705 \& 2020YFB2103705) and Shenzhen Postdoctoral Research Fund (Grant Number: K21627501).

\section{References}

[1] Mohamad M, Selamat A, Krejcar O, Fujita H and Wu T 2020 An analysis on new hybrid parameter selection model performance over big data set Knowledge-Based Systems 192105441.

[2] Xiao X and Xie C 2021 Rational planning and urban governance based on smart cities and big data Environmental Technology \& Innovation 21101381.

[3] Kandt J and Batty M 2021 Smart cities, big data and urban policy: Towards urban analytics for the long run Cities 109102992.

[4] Liu L 2021 Green urban environmental sustainability and health sport based on MapReduce fitness big data and ZigBee technology Environmental Technology \& Innovation 23101676.

[5] Ramírez T, Hurtubia R, Lobel H and Rossetti T 2021 Measuring heterogeneous perception of urban space with massive data and machine learning: An application to safety Landscape and Urban Planning $\mathbf{2 0 8}$ 104002.

[6] Chen W 2018 Architectural style analysis method based on intelligent computing technology 2018 10th International Conference on Measuring Technology and Mechatronics Automation (ICMTMA) pp 321324.

[7] Bao J, Yang Z, Zeng W and Shi X 2021 Exploring the spatial impacts of human activities on urban traffic crashes using multi-source big data Journal of Transport Geography 94103118.

[8] Lei Z, Chen Y and Lim M K 2021 Modelling and analysis of big data platform group adoption behaviour based on social network analysis Technology in Society 65101570.

[9] Salloum S, Huang J Z and He Y 2019 Random sample partition: A distributed data model for big data analysis IEEE Transactions on Industrial Informatics 15 5846-5854.

[10] Luo J, Zu X F, Chen L and Du W 2012 Design of data sharing and exchange interface of telecom enterprise marketing management System IERI Procedia 13907. 